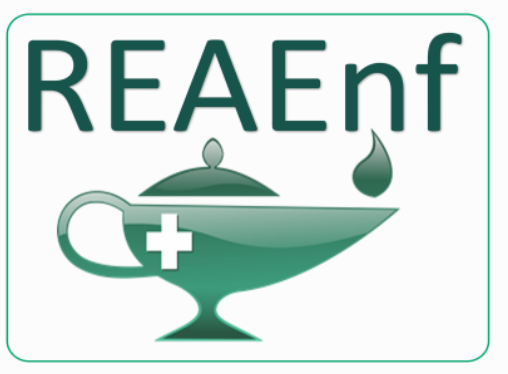

Revista Eletrônica Acervo Enfermagem
REVISÃO BIBLIOGRÁFICA

Recebido em: 8/2020

Aceito em: 9/2020

Publicado em: 1/2021

\title{
O papel do enfermeiro frente a violência infantil na atenção primária
}

\author{
The role of nurse in front of child violence in primary care
}

El papel de las enfermeras frente a la violencia infantil em la atención primaria

Stefany Alves da Silva ${ }^{1 *}$, Carina Ceribelli².

\begin{abstract}
Resumo: A violência infantil é uma realidade crescente no Brasil se tornando um grave problema de saúde pública. A atenção primária se torna o ambiente ideal para o enfrentamento dessa temática devido o vínculo entre profissional e a comunidade, tendo o enfermeiro um papel fundamental para intervir no ciclo da violência e não permitir a sua perpetuação. Entretanto, nota-se que o enfermeiro não sabe o seu papel frente esta temática, se colocando muitas vezes como incapaz de intervir frente a esses casos, portanto o objetivo deste artigo é estudar o papel do enfermeiro frente a violência e vitimização infantil na atenção primária através de revisão bibliográfica. O estudo permitiu se evidenciar três principais papeis do enfermeiro frente a esses casos na atenção primária, sendo eles: identificação dos casos, notificação compulsória e prevenção e promoção. Contudo, se evidenciou que os profissionais enfrentam muitos limites e desafios se mostrando como os principais a ausência de formação e capacitação, medo e ausência de fluxograma de apoio. Assim, se concluindo a relevância da abordagem da temática nas grades curriculares do curso de enfermagem e a necessidade de uma rede de apoio organizada e articulada para enfrentamento destes casos.
\end{abstract}

Palavras-chave: Enfermeiro, Violência infantil, Atenção primária.

\begin{abstract}
Child violence is a growing reality in Brazil, becoming a serious public health problem. Primary care becomes the ideal environment to face this theme due to the bond between professional and the community, with nurses having a fundamental role to intervene in the cycle of violence and not allow its perpetuation. However, it is noted that nurses do not know their role in relation to this topic, often placing themselves as incapable of intervening in these cases, so the objective of this article is to study the role of nurses in the face of child violence and victimization in primary care through critical bibliographic review of articles. The study made it possible to highlight three main roles of nurses in relation to these cases in primary care, namely: case identification, compulsory notification and prevention and promotion. However, it became evident that the professionals face many limits and challenges, showing themselves as the main ones the absence of training and qualification, fear and absence of support flowchart. Thus, concluding the relevance of the approach of the theme in the curriculum of the nursing course and the need for an organized and articulated support network to face these cases.
\end{abstract}

Keywords: Nurse, Child Violence, Primary attention.

${ }^{1}$ Faculdades Metropolitanas Unidas (FMU), São Paulo-SP. *E-mail: stee.enfer@gmail.com

Escola de Enfermagem de Ribeirão Preto da Universidade de São Paulo (EERP-USP), Ribeirão Preto-SP. 
Resumen: La violencia infantil es una realidad creciente en Brasil, convirtiéndose en un grave problema de salud pública. La atención primaria se convierte en el entorno idóneo para afrontar esta temática por el vínculo entre el profesional y la comunidad, teniendo el enfermero un papel fundamental para intervenir en el ciclo de la violencia y no permitir su perpetuación. Sin embargo, se observa que las enfermeras desconocen su rol en relación a este tema, ubicándose muchas veces como incapaces de intervenir en estos casos, por lo que el objetivo de este artículo es estudiar el papel de las enfermeras ante la violencia y victimización infantil en la atención primaria. Mediante revisión bibliográfica crítica de artículos. El estudio permitió destacar tres roles principales del enfermero en relación con estos casos en atención primaria, a saber: identificación de casos, notificación obligatoria y prevención y promoción. Sin embargo, se hizo evidente que los profesionales enfrentan muchos límites y desafíos, mostrándose como los principales la ausencia de formación y calificación, el miedo y la ausencia de diagrama de flujo de apoyo. Se concluye así la relevancia del abordaje del tema en el currículo del curso de enfermería y la necesidad de una red de apoyo organizada y articulada para enfrentar estos casos.

Palabras clave: Enfermera, Violencia infantil, Atención primaria.

\section{INTRODUÇÃO}

Segundo a Organização Mundial de Saúde (2012), a violência é o uso deliberado da força física, podendo ser contra si próprio ou contra terceiros podendo ocasionar lesões físicas, óbito e danos psicossociais.

A violência atualmente é algo expressivo no cotidiano, estando enraizada na trajetória da humanidade. Desde a pré-história ela está presente sendo utilizada como meio de sobrevivência do homem, contudo, com a evolução da sociedade a violência foi adquirindo novas formas e funções e passou a ser uma ferramenta de poder utilizada sobre os mais vulneráveis para se obter algo (ZOTTIS GAH, et al., 2006).

O comportamento violento frequentemente é caracterizado como um ciclo, onde os indivíduos que passarão por experiencias violentas na infância, tendem a praticar a violência em seus próprios relacionamentos, pois, a criança utiliza o modelo referencial adulto para formar seus parâmetros comportamentais, assim naturalizando o comportamento violento e o reproduzindo. Este fenômeno, é conhecido como violência intergeracional (ABRANCHES CD e ASSIS SGA, 2011).

Existem outras particularidades que corroboram para predisposição da violência infantil, como: toxicodependência, dificuldade no vínculo com a criança, perturbação física ou mental na criança, crianças hiperativas, gravidez indesejada, múltiplos filhos, família monoparental, baixa condição socioeconômica, conflitos conjugais, valores culturais e religiosos, precariedade de serviços sociais, condições de habitação e baixa escolaridade (CAMPOS SIF, 2010).

As crianças possuem maior vulnerabilidade a violência, por estarem em fase de desenvolvimento psicossocial pode acarretar graves problemas emocionais, psicológicos, sociais e cognitivos, com consequências na saúde da criança ao longo de sua vida. Quando praticada no contexto intrafamiliar pode levar a vítima a tornasse mais suscetíveis a violência em outros ambientes sociais e nas relações (ABRANCHES CD e ASSIS SGA, 2011).

A violência pode se apresentar de diversas formas, sendo suas principais: a física, negligência, violência psicológica e violência sexual (MAGALHÃES MLC, et al., 2009).

A violência física é o ato de se empregar força física de forma intencional podendo provocar ou não danos físicos. Essa variável é frequentemente justificada pelos pais ou cuidadores como forma de educar (MINISTÉRIO DA SAÚDE, 2015). Entretanto, o uso castigo físico como forma de disciplinar se tornou crime no Brasil desde 2014 conforme a Lei $n^{\circ} 13.010$, conhecida popularmente como "lei da palmada".

Já a negligência é caracterizada pela omissão dos cuidadores de prover as necessidades básicas da criança para o seu desenvolvimento saudável, podendo se apresentar como privação de cuidados com a 
saúde, ausência de estímulos, descuido com a higiene e abandono temporário ou permanente. Pode ser classificada como negligência ativa onde existe intenção de dano e negligência passiva onde os responsáveis têm a incapacidade de prover as necessidades básicas da criança (CARVALHO BL, 2018).

Se considera violência psicológica atos e palavras que podem provocar danos ao desenvolvimento emocional da criança, por não deixar sinais visíveis é de difícil detecção, entretanto, pode marcar a criança gerando consequências na vida adulta como baixa autoestima (MAGALHÃES MLC, et al., 2009).

Se compreende violência sexual, qualquer ato sexual que seja imposto a uma criança por um agressor com estado psicossexual superior a mesma. Possuindo diversas facetas, desde o exibicionismo até a consumação do ato sexual (ZOTTIS GAH, et al., 2006).

A Constituição Federal de 1988 e o Estatuto da Criança e do Adolescente (ECA), estabelecem proteção integral à criança e o adolescente zelando pelos seus direitos. Contudo, os dados epidemiológicos mostram um crescente aumento da violência infantil, de acordo com o Sistema de Informações de Agravos e Notificações (SINAN) no ano que 2011 foram computadorizados 39.281 atendimentos entre a faixa etária de 1 a 19 anos, sendo $40,5 \%$ de violência física, seguido de $19,9 \%$ abuso sexual, $17 \%$ violência psicológica, $15,8 \%$ negligência ou abandono e $6,8 \%$ outros tipos de violência. Contudo, no ano de 2017 o SINAN registrou 31.435 notificações, referente apenas a violência sexual contra crianças e adolescentes (BRASIL, 2015). Portanto, se caracteriza como um grave problema de saúde pública devido grande demanda de atendimentos nos serviços de públicos de saúde.

As Unidades Básicas de Saúde (UBS), se caracterizam como o ambiente ideal para o enfrentamento deste fenômeno, devido ao vínculo estabelecido entre profissional e paciente, podendo proporcionar uma prevenção ou detecção precoce (SANTOS JS e YAKUWA MS, 2015).

Mediante as colocações aqui elencadas, notasse que o presente tema se configura como um desafio devido a sua complexidade. Tendo o enfermeiro um papel essencial, sobretudo aqueles que atuam na atenção primária, pois são porta do serviço de saúde e conseguem identificar casos de violência infantil e fatores de risco e podendo desenvolver medidas de prevenção com mais facilidade. No entanto percebese através da revisão bibliográfica que o enfermeiro não sabe seu papel frente temática, se colocando na maioria das vezes como incapaz de intervir. Portanto, este estudo tem como objetivo identificar o papel do enfermeiro frente a violência infantil na atenção primária.

\section{REVISÃO BIBLIOGRÁFICA}

\section{Identificação e notificação compulsória dos casos de violência infantil}

Todos os artigos selecionados corroboram que é função do enfermeiro a identificação, investigação e notificação dos casos suspeitos ou confirmados de violência infantil.

As unidades de atenção primária á saúde são consideradas a base do sistema de saúde, sendo a porta de entrada dos usuários ao serviço de saúde público, onde os indivíduos são acompanhados ao longo de toda a sua vida, portanto, é o ambiente ideal para identificação de fatores de risco para os casos de violência infantil. Entretanto, para se fazer a identificação e investigação de casos e fatores de risco é necessário que o profissional seja capacitado para notar os sinais indiretos que a criança pode vir a apresentar que indicam presença de violência (PAIXÃO GNT, et al., 2013).

Schwanck HA, et al. (2005), realizou um estudo cujo o objetivo era averiguar a compreensão dos discentes do sétimo semestre da graduação em enfermagem de uma universidade acerca da sua preparação para reconhecer e cuidar de crianças vítimas de violência infantil, onde notou-se que metade dos acadêmicos não se sentiam seguros para atuarem nesses casos, pois não haviam preparo suficiente.

Outro estudo realizado por Dias EP, et al. (2013), com enfermeiros de UBS's no município de Uberlândia - MG, evidenciou que todos os enfermeiros não tiveram ou não se lembraram de terem tido disciplina que abordassem esta temática durante a graduação. Assim, evidenciando a carência da temática nos currículos de graduação. 
Portanto, se faz indispensável que as instituições de atenção desenvolvam ações de capacitação para os profissionais, devido a dificuldades dos mesmos em identificar e conduzir os casos relacionados a violência infantil, pela carência de conhecimento sobre a temática derivado de uma formação acadêmica deficiente (MELO RA, et al., 2016; PIRES AC, 2017). No que se refere aos enfermeiros capacitados, devido ao cotidiano cheio demandas acaba-se negligenciando os sinais apresentados pela criança durante a consulta de enfermagem, sendo necessário que o enfermeiro inclua espaço em sua rotina para uma anamnese durante as consultas em busca desse problema (THOMAZINE AM, et al., 2009).

A notificação dos casos suspeitos e confirmados de violência infantil é de caráter compulsório nos serviços de saúde públicos e privados através da portaria $n^{\circ}$ 1.968/2001 (BRASIL, 2001). Além disso, quando o profissional de saúde realiza a notificação, ele está agindo de acordo com o Código de Ética de Enfermagem que estabelece como proibição ser conivente ou omisso a qualquer forma de violência durante o exercício da profissão, sendo sujeito a medidas disciplinares conforme gravidade do caso. $O$ ECA ainda estabelece em seu Art. 245, os profissionais que são obrigatórios realizarem a notificação se enquadrando entre eles os profissionais de saúde, ficando sujeito aos profissionais que não realizarem a notificação multa de vinte e três salários de referência, sendo estabelecido o dobro desse valor caso de reincidência (BRASIL, 1996).

Segundo Paixão GNP, et al. (2013), é incontestável a existente de uma lacuna nas notificações dos casos de violência infantil. Observa-se que na maioria dos artigos selecionados os profissionais de saúde veem a notificação como ferramenta importante para a descontinuidade da violência, entretanto, os mesmo relatam a relutância em adotarem a notificação como conduta a ser tomada, sendo justificado pela carência de qualificação que faz com que o profissional se sinta desqualificado para executar a mesma, medo de represarias advindas dos agressores e dificuldade de identificação dos casos (BEZERRA KP, et al., 2012; THOMAZINE AM, et al., 2014; MAIA JN, et al., 2016; MELO RA, et al., 2016).

Deve-se se enfatizar que através dos dados que a notificação fornece é possível se formar um indicador epidemiológico da violência infantil, assim redirecionando verbas e criando políticas para proteção e defesa dos direitos das crianças e dos adolescentes. É importante ressaltar que a notificação é apenas o primeiro passo para a proteção da vítima, devendo o enfermeiro junto a equipe multidisciplinar realizar um planejamento contínuo de cuidados e estratégias para minimizar o trauma sofrido e reincidências (PAIXÃO GPN, et al., 2013; THOMAZINE AM, et al., 2014).

\section{Promoção e prevenção}

É atribuição do enfermeiro promover a educação em saúde visando a melhoria da saúde da população conforme a Lei $n^{\circ} 7.498 / 1986$ que dispõe sobre a regulamentação do exercício da profissão de enfermagem. Sendo, a educação em saúde um dos pilares da atenção primária, visando a promoção e prevenção em saúde (BRASIL, 1986).

Os estudos evidenciam que as diferentes formas de violência podem ser prevenidas, como qualquer agravo de saúde. Contudo, é preciso estabelecer ações que visam o acompanhamento e reconhecimento do problema, identificação de grupos e fatores de vulnerabilidade para que assim sejam implantadas ações focadas nesse público alvo (IOSSI MA, 2004; DIAS EP, 2012). Neste contexto, o enfermeiro deve realizar o papel de educador buscando trabalhar com a comunidade a ideologia da proteção da criança e do adolescente, através de palestras, oficinas, constelação familiar e programas educativos a famílias em situação de risco.

Os enfermeiros através das consultas de enfermagem e realização de visitas domiciliares tem a chance de conhecer o contexto familiar de forma intima, desencadeando um ambiente propicio para detecção precoce dos fatores de risco e medidas preventivas, dessa forma criando mecanismos para evitar a perpetuação da violência (ALGERI S, e SOUZA LM, 2006; PIRES AC, 2017).

Autores como Caminha RM (2004) e Grudtener DI (2005) e Algeri S e Souza LM (2006) seguem uma linha de raciocínio para a prevenção dos casos de violência infantil, onde a prevenção deve ocorrer nos três níveis da assistência. Entretanto, se destaca o modelo de Algeri S e Souza LM (2006); que aborda 
diretamente as intervenções dos profissionais de enfermagem nos três níveis da assistência a saúde na atenção primária, visando criar um mecanismo para nortear as ações que devem ser estabelecidas.

Segundo Algeri S e Souza LM (2006), cabe a atenção primária ações educativas que frisem a proteção da criança e do adolescente. Podendo se estabelecer ações como palestras que visem transmitir formas de lidar com conflitos familiares e métodos alternativos de se educar, intervenções durante o pré-natal para fortalecimento do vínculo com a criança e até mesmo o planejamento familiar como forma de prevenir gravidez indesejada, tendo todas essas ações o objeto de diminuir a incidência e prevalência da violência infantil (PAIXÃO GPN, et al., 2013; PIRES AC, 2017).

Em relação a atenção secundária segundo Algeri S e Souza LM (2006), o enfermeiro deve focar na detecção de famílias que apresentem fatores de risco para a violência, devendo se avaliar estes fatores principalmente nas consultas pré-natais e puérperas, orientando sobre a importância do disciplinamento positivo o diferenciando da rigidez e omissão e acompanhar a evolução da dinâmica familiar em visitas domiciliares. Nos casos de suspeita de violência deve-se fazer a investigação junto a equipe multidisciplinar e realização da notificação (PAIXÃO GPN, et al., 2013; DIAS EP, et al., 2013; PIRES AC, 2017).

No que desrespeito a atenção terciaria Algeri S e Souza LM (2006), traz como função do enfermeiro o acompanhamento da vítima e sua família, abordado junto a equipe multidisciplinar a criação de um plano de cuidados, visando minimizar os traumas sofridos, evitar a reincidência e restabelecer a dinâmica famíliar. Contudo, nos casos mais graves onde se faz necessário o afastamento da criança de sua família cabe ao enfermeiro acompanhar o caso através da ferramenta de contra-referência (PAIXÃO GPN, et al., 2013; PIRES AC, 2017).

\section{Limites e desafios da assistência de enfermagem}

O medo foi evidenciado na maioria dos artigos selecionados como um dos principais obstáculos para a assistência de enfermagem. Sendo, um fator que influencia nas ações que serão tomadas levando na maioria dos casos a realização de subnotificações e negligência na prestação dos cuidados negando a devida atenção que a temática exige, acarretando na carência de medidas preventivas para evitar incidência e reincidência (BEZERRA KP e MONTEIRO AI, 2012; LUSTOSA AP, et al., 2014).

Em relação as equipes das UBS's por atuarem diretamente com a comunidade, possibilita a criação de um vínculo profissional-paciente, se tornando um ambiente favorável para a identificação precoce de fatores de risco, investigação de casos suspeitos e medidas preventivas. Entretanto, está mesma aproximação e a ausência de leis vigentes de proteção ao profissional, levam o mesmo a se sentir desprotegido para lidar com estes casos (BEZERRA KP e MONTEIRO AI, 2012; LUSTOSA AP, et al., 2014; MAIA JN, et al., 2016).

Nota-se nos artigos o relato da falta de credibilidade na rede de proteção à criança, ausência de serviços de referência e a não resolutividade dos casos encaminhados, como fatores que levam a subnotificações. Fazendo-se necessário uma intersetorialidade bem estruturada e organizada, para que todos saibam suas responsabilidades e funções, para que os encaminhamentos sejam resolutivos (SANTOS JA e YAKUMA MS, 2015; MAIA JN, et al., 2016; MELO RA, et al., 2016).

Em relação a notificação autores como Ribeiro MA, et al. (2004), Silva MAl e Ferriani MGC (2007), Guimarães JATL e Villela WN (2011), observam a baixa adesão do profissional de enfermagem na realização de notificação de casos suspeitos e confirmados, sendo justificada por alguns autores por carência de qualificação profissional para detecção de casos e medo de identificar-se devido as consequências que tal pratica pode vir a acarretar.

Nos estudos analisados, foi quase unânime que os enfermeiros têm dificuldade de identificar e conduzir os casos relacionados a esta temática. Sendo justificada pelo fato da formação biomédica e ausência da temática na formação dos profissionais levando ao despreparo para lidar com esses casos, se fazendo necessário a inclusão da temática nas grades curriculares das graduações de enfermagem (THOMAZINE AM, et al., 2014; MAIA JN, et al., 2016; MELO RA, et al., 2016; PIRES AC, 2017). 
A atenção primária é o ambiente mais propicio para identificação e prevenção de casos de violência infantil. Portanto, a capacitação dos profissionais que atuam neste setor é essencial, devendo-se enfatizar o conhecimento das políticas que guardam os direitos das crianças e adolescentes, bem como a rede de proteção para as vítimas (ARAGÃO AS, et al., 2013).

Ao se analisar os artigos, evidenciou-se que em apenas um estudo realizado por Leite JT, et al. (2016) foi relatado o conhecimento de ações da Secretaria de Saúde para a capacitação dos enfermeiros sobre a temática. Entretanto, é relatado a ausência nesses eventos devido a falta de funcionários e sobrecarga de trabalho (SÁ CMQ, et al., 2016).

Relata-se a dificuldade dos profissionais em lidar com os casos de violência infantil devido a questão psicológica envolvida, sendo necessário um olhar do enfermeiro para preparar o emocional e psicológico da sua equipe para estarem aptos para lidar com esses casos (MELO RA, et al., 2016; LEITE JT, et al., 2016; PIRES AC, 2017).

A ausência de contra referência dos casos encaminhados para o conselho tutelar ou rede de proteção a criança é uma dificuldade, levando a vítima e seus familiares fiquem desassistidos. Portanto, não podendo se fazer um plano de intervenções para a prevenção de reincidência, melhora da dinâmica familiar e minimização dos traumas causados (SÁ CMQ, et al., 2016).

\section{CONSIDERAÇÕES FINAIS}

É de responsabilidade do enfermeiro a notificação dos casos suspeitos ou confirmados de violência infantil, identificação e detecção de violência infantil, educação da população quanto a ideologia de proteção a criança, intervenções em fatores de riscos para violência infantil e fornece suporte e treinamento da sua equipe para enfrentamento desses casos. Se fazendo necessário a inclusão da temática nas grades curriculares, implantação de ações de educação permanente e de protocolos que guiem os encaminhamentos e intervenções dos profissionais de enfermagem frente a estes casos.

\section{REFERÊNCIAS}

1. ABRANCHES CD, ASSIS SG. A (in)visibilidade da violência psicológica na infância e adolescência no contexto familiar. Caderno de Saúde Pública, 2011; 27(5): 843-854.

2. ALGERI S, SOUZA LM. Violência contra crianças e adolescentes: um desafio no cotidiano da equipe de enfermagem. Revista Latino Americana de Enfermagem, 2006; 14(4): 625-631.

3. ARAGÃO AS, et al. Abordagem dos casos de violência à criança pela enfermagem na atenção básica. Revista Latino Americana de Enfermagem, 2013; 21 (7), 1-7.

4. BEZERRA KP, MONTEIRO Al. Violência intrafamiliar contra a criança: intervenção de enfermeiros da Estratégia e Saúde da Família. Revista Rene, 2012, 13(2): 354-364.

5. BRASIL. Lei n ${ }^{\circ}$ 1.968, 25 de outubro de 2001. Dispõe sobre a notificação, ás autoridades competentes, de casos de suspeita ou de confirmação de maus-tratos contra crianças e adolescentes atendidos nas entidades do SUS. Brasília: casa civil, 2001.

6. BRASIL. Lei $n^{\circ} 7.498,25$ de junho de 1986. Dispõe sobre a regulamentação do exercício da enfermagem, e dá outras providências. Brasília: casa civil, 1986.

7. BRASIL. Lei n 8.394, 20 de dezembro de 1996. Dispõe sobre o Estatuto da Criança e do Adolescente e dá outras providências. Brasília: casa cibil, 1996.

8. BRASIL. Ministério da Saúde. Secretaria de Vigilância em Saúde. Análise Epidemiológica da Violência Sexual contra Crianças e Adolescentes no Brasil, 2011 a 2017, 2010; 49 (27).

9. CARVALHO BL. Papel do Enfermeiro nos Maus Tatos / Negligência das Crianças - Preocupação Contínua. Dissertação (Mestrado em Enfermagem) - Escola Superior de Saúde de Santarém, 2018; 156 p.

10. CAMINHA RM, et al. A Epidemiologia de abuso sexual infantil na clínica escola PIPAS/UNISNOS. Cadernos de Extensão da UNINOS/RS, 2005; 4: 129-142.

11. CAMPOS SIF. O Tabu da Violência Intrafamiliar: a escola na fronteira. SINAIS revista eletrônica, 2010; 1(7): 145174.

12. DIAS EP. Enfermeiro no Atendimento de Casos de Violência Doméstica Infantil em Unidades Básicas de Saúde Uberlândia - SP. Revista LEVS UNESP, 2012; 12: 118-135. 
13. GRUDTNER DI. Violência intrafamiliar contra a criança e o adolescente: reflexões sobre o cuidado de enfermeira. Texto Contexto - Enfermagem, 2005, 16 (1): 182-183.

14. GUIMARÃES JATL, VILLELA WV. Características da violência física e sexual contra crianças e adolescentes atendidos no IML de Maceió, Alagoas, Brasil. Cadernos de Saúde Pública, 2011; 27(8): 1647- 1653.

15. IOSSI MA. O envolvimento dos profissionais da saúde no município de Guarulhos na assistência ás crianças vítimas de violência doméstica: um caminhas necessário. Dissertação (Doutorado em Enfermagem) - Escola de Enfermagem de Ribeirão Preto, Universidade de São Paulo, Ribeirão Preto, 2004; 143 p.

16. LEITE JT. Enfrentamento da violência doméstica contra crianças e adolescente na perspectiva de enfermeiros da atenção básica. Revista Gaúcha de Enfermagem - online, 2016; 37(2): 1-7.

17. LUSTOSA AP, et al. Abuso sexual contra crianças: evidências para o cuidado de Enfermagem. Caderno ESP, $2014 ; 8(2): 50-63$.

18. MAGALHÃES MLC, et al. O profissional de saúde e a violência na infância e adolescência. Revista Feminina, 2009; 37(10): 548-551.

19. MAIA JN, et al. Violência contra criança: cotidiano de profissionais na atenção primária a saúde. Revista Multidisciplinar e de Psicologia, 2016; 10(32): 345-359.

20. ORGANIZAÇÃO MUNDIAL DA SAÚDE. Relatório Mundial sobre Violência e Saúde. Gebenebra, 2002.

21. PAIXÃO GPN, et al. Violência Intrafamiliar contra criança: atribuições do profissional de enfermagem. Revista Eletrônica da Fainor, 2013; 6(2): 22-39.

22. PIRES AC. Papel do enfermeiro frente ao abuso sexual de crianças e adolescentes. Dissertação (Graduação) Faculdade de Enfermagem, Centro Universitário de Brasília, 2017; 21 p.

23. RIBEIRO MA, et al. Violência sexual contra crianças e adolescentes: características relativas a vitimização nas relações familiares. Cadernos de Saúde Pública, 2001; 20(4): 456-464.

24. SÁ $C M Q$, et al. Atenção da equipe de enfermagem frente á violência sexual contra crianças e adolescentes. Mostra Interdisciplinar do Curso de Enfermagem, 2016; 2(2).

25. SANTOS JS, YAKUWA MS. A Estratégia Saúde da Família frente a Violência contra Crianças: revisão integrativa. Revista Sociedade Brasileira de Enfermagem Pediatrica, 2015; 15(1): 38-43.

26. SCHWANCK RH, et al. A Percepção de Formandos de Enfermagem Acerca da Violência Contra a Criança. Cogitare Enfermagem, 2005; 10(2): 41-46.

27. SILVA MIA, FERRIANI MGC. Violência Doméstica: do visível ao invisível. Revista Latino Americana de Enfermagem, 2007; 15(2): 1-8.

28. THOMAZINE AM, et al. Atenção a crianças e adolescentes vítimas de violência intrafamiliar por enfermeiros em serviços de pronto atendimento. Revista Eletrônica de Enfermagem, 2009; 11(4): 830-840.

29. THOMAZINE AM, et al. Abordagem da atenção primária á saúde na violência intrafamiliar infantil. Revista da Sociedade Brasileira de Enfermeiros Pediatras, 2004; 14(2): 105-112.

30. ZOTTIS GAH, et al. Violência Intrafamiliar Contra a Criança e as Atribuições do Profissional de Enfermagem. Dm. Saúde e Desenvolvimento, 2006; 8(2): 146-153. 